\title{
Sarcoma de Kaposi em paciente portador de HIV: relato de caso
}

\author{
Kaposi sarcoma in a patient with HIV: case report
}

Amanda Kloster Wagner ${ }^{1}$, Jéssica Priscila Tozo ${ }^{1}$, Marilda Aparecida Mllanez Morgado de Abreu², Glaucia Ferreira Wedy ${ }^{3}$

\begin{abstract}
RESUMO
Sarcoma de Kaposi é um tumor maligno originado do endotélio vascular que acomete principalmente pele e mucosas. Geralmente, é associado à síndrome da imunodeficiência adquirida - aids, apresentando lesões vinhosas, arredondadas que, com o passar dos dias, tornam-se purpúricas, elevadas e com distribuição multifocal. Nesse estudo é relatado o caso de um paciente do sexo masculino, de 42 anos de idade, de fototipo IV, com emagrecimento, diarreia, pápulas e placas eritemato-violáceas nos membros inferiores. Durante a internação, encontrou-se sorologia positiva para HIV e ao realizar histopatológico das lesões cutâneas, confirmou-se o diagnóstico de sarcoma de Kaposi. O objetivo do presente estudo é ressaltar que quando presente infecção pelo citomegalovírus em paciente com aids há maior predisposição para o desenvolvimento de tal neoplasia.
\end{abstract}

Palavras chave: Sarcoma de Kaposi. Herpesvirus Humano 8. Sorodiagnóstico da AIDS.

\begin{abstract}
Kaposi's sarcoma is a malignant tumor originating from the vascular endothelium, which mainly affects the skin and mucous membranes. Generally, it is associated with acquired immunodeficiency syndrome - AIDS, presenting rounded, wine-like lesions that become purpuric, elevated, and multifocal in the course of days. In this study the case of a 42-year-old male phototype IV with weight loss, diarrhea, papules and erythematous-purple plaques in the lower limbs was reported. During the hospitalization, positive serology for HIV was found and the diagnosis of Kaposi's sarcoma was confirmed in the histopathological examination of cutaneous lesions. The objective of the present study is to highlight that when present with cytomegalovirus in a patient with AIDS there is a greater predisposition for the development of such neoplasia.
\end{abstract}

Keyword: Sarcoma, Kaposi. Herpesvirus 8, Human. AIDS Serodiagnosis.

1. Discente, estudante de Medicina, Universidade do Oeste Paulista

2. Docente, Disciplina de Dermatologia da UNOESTE; Chefe do Serviço de Dermatologia do Hospital Regional de Presidente Prudente/UNOESTE.

3. Médica especializada em Dermatologia no serviço de Dermatologia do Hospital Regional de Presidente Prudente.
CoRRESPONDÊNCIA: Serviço de Dermatologia do Hospital Regional de Presidente Prudente/UNOESTE. Rua São Paulo, 1949 bairro centro CEP 17900-000 Dracena-SP.

Recebido em 07/06/2017 Aprovado em 28/10/2017 


\section{INTRODUÇÃO}

O sarcoma de Kaposi foi descrito pela primeira vez na literatura em 1872 por Moritz Kaposi, com o nome de sarcoma múltiplo pigmentado idiopático. ${ }^{1}$ Tem origem no endotélio vascular, configurando-se em uma doença sistêmica de variável grau de malignidade. ${ }^{2}$

Sua etiologia não é claramente explicada. Sabe-se que existem vários fatores envolvidos e que há relação com a infecção pelo vírus da imunodeficiência humana (HIV) e herpes vírus humano-8 (HHV-8). O HHV-8 apresenta homólogos de genes celulares que estimulam a proliferação de células, inflamação e angiogênese. Outros fatores envolvidos na gênese da doença são gênero, imunossupressão, predisposição genética e ativação de citocinas. ${ }^{2}$

É mais comum em pacientes masculinos, na faixa etária mais avançada, com ascendência mediterrânea. ${ }^{2}$

A evolução clínica é extremamente variável. Na maioria segue um curso benigno, longo e indolente e, embora progressivo, tende a permanecer confinado à pele e aos tecidos subcutâneos. ${ }^{1}$ Quando mais grave, pode acometer vísceras, como tubo digestório e pulmão, além de mucosas e, especialmente, a pele, órgão mais afetado, no qual se manifesta na forma de manchas, pápulas, placas ou lesões nodulares profundas, multifocais, castanho-violáceas ou vermelho-escuras, localizadas principalmente nos membros inferiores, raramente comprometendo vísceras. ${ }^{3,4}$ Essas lesões podem sangrar, ulcerar e estar asscociadas a linfedema, dor e infecção secundária. ${ }^{1}$

No que se refere à classificação, o sarcoma de Kaposi pode ser: clássico, endêmico, iatrogênico e epidêmico, sendo o último associado à síndrome da imunodeficiência adquirida - aids. ${ }^{5} \mathrm{~A}$ forma clássica manifesta-se, especialmente, em homens idosos e na população descendente de judeus do leste europeu, sob a forma cutânea, principalmente nos membros inferiores; enquanto a forma endêmica prevalece na população africana, com semelhança à forma clássica, sendo mais agressiva na população mais jovem. A forma iatrogênica se expressa em pacientes que estão em uso de drogas ou condições que levem a imunossupressão, sendo o gênero masculino, com idade variável, o mais suscetível de acometimento. Já, a forma epidêmica está relacionada à infecção por HIV, principalmente em homens, na faixa etária entre 18-65 anos. ${ }^{1,4}$

Segundo a literatura, os portadores de HIV têm risco 3.640 vezes maior de adquirir sarcoma de Kaposi do que a população em geral. Desta forma, a infecção é um parâmetro importante para o diagnóstico. Quando associado à aids, o sarcoma de Kaposi manifesta-se de forma mais agressiva, surgindo lesões na pele e nas vísceras com progressão variável. ${ }^{4,5}$

O diagnóstico fundamenta-se no exame físico completo do paciente, incluindo membranas mucosas da cavidade oral e genital e, no exame anatomopatológico da lesão suspeita, além de exames complementares a fim de determinar o estádio da doença, dentre eles: raio $\mathrm{x}$ simples do tórax, estudo da subpopulação de linfócitos e da carga viral, ecografia de linfonodos e abdominal, endoscopia digestiva alta e colonoscopia. 4,6 Uma vez diagnosticada a doença, o tratamento varia de acordo com a gravidade, tamanho e localização das lesões, podendo nos casos localizados ser realizada a remoção cirúrgica, e nos mais disseminados, radioterapia e quimioterapia. ${ }^{7}$

O Citomegalovírus (CMV) é um vírus oportunista com material genético constituído por DNA da família herpesvirus, que causa grande morbidade e mortalidade em paciente com HIV. ${ }^{8}$ Pacientes podem ter alterações pulmonares, gastrintestinais ou do sistema nervoso central. $\mathrm{Na}$ fase terminal da aids, a infecção por CMV causa retinite em até $40 \%$ dos pacientes e anormalidades da retina visíveis ao fundo de olho e podem causar doença ulcerativa do cólon e do esôfago. ${ }^{8,9}$

Pode permanecer no organismo indefinidamente após a primeira infecção e, em determinadas situações de baixa imunidade do hospedeiro, o vírus pode ser ativado e causar infecções recorrentes. Em indivíduos imunocompetentes geralmente estabelece uma infecção primária assintomática com baixos ou nenhum nível de replicação viral. No entanto, em indivíduos infectados pelo HIV, o risco de doenças sintomáticas aumenta com o grau de imunossupressão. ${ }^{9}$

Além das inúmeras complicações, a infecção pelo CMV na sua forma ativa, pode alterar o 
tropismo celular do HIV, o que facilita a entrada do material genético do HIV nos fibroblastos humanos, normalmente não permissíveis. Já o HIV provoca uma progressiva deficiência no sistema imunológico, permitindo que o CMV seja reativado em pessoas infectadas que possuem níveis baixos de linfócitos T CD4+. Esse aumento da replicação do HIV, resulta no aumento da replicação do CMV, o que cria um ciclo vicioso e acelera a progressão da aids. ${ }^{9,10}$

\section{RELATO DE CASO}

Paciente masculino, 42 anos, fototipo IV, com quadro de diarreia mucopiossanguinolenta, emagrecimento e lesões cutâneas nos membros inferiores há dois meses. Sorologia para anticorpos contra HIV reagente. O quadro diarreico teve como causa retocolite provocada pelo CMV cujo diagnóstico através da colonoscopia + biopsia das lesões macroscópicas. Referia episódio de herpes zoster na face há 3 meses.

Apresentava ao exame dermatológico, placas e pápulas eritematoacastanhadas infiltrativas, descamativas, não pruriginosas, com diâmetros variáveis (Figura 1, 2 e 3). Os exames anatomopatológico e imuno-histoquímico revelaram, respectivamente, lesão proliferativa vascular e expressão para células endoteliais (CD34 e CD31), apresentando perfil morfológico e imuno-histoquímico compatível com sarcoma de Kaposi.

O paciente segue em acompanhamento, com melhora do quadro após ser submetido à radioterapia e quimioterapia com vincristina e terapia antirretroviral.

\section{DISCUSSÃO}

O sarcoma de Kaposi é uma das neoplasias mais comuns no paciente com aids, além de ser uma doença definidora da imunodeficiência grave. Pode acometer pele, mucosas e órgãos internos. Sua evolução clínica varia entre os pacientes, desde lesões indolentes até um comportamento agressivo com graves complicações. ${ }^{11}$

Normalmente, as lesões começam como manchas avermelhadas, pálidas, que com passar dos dias tornam-se purpúricas, elevadas, arredondadas, podendo variar de milímetros a centí-

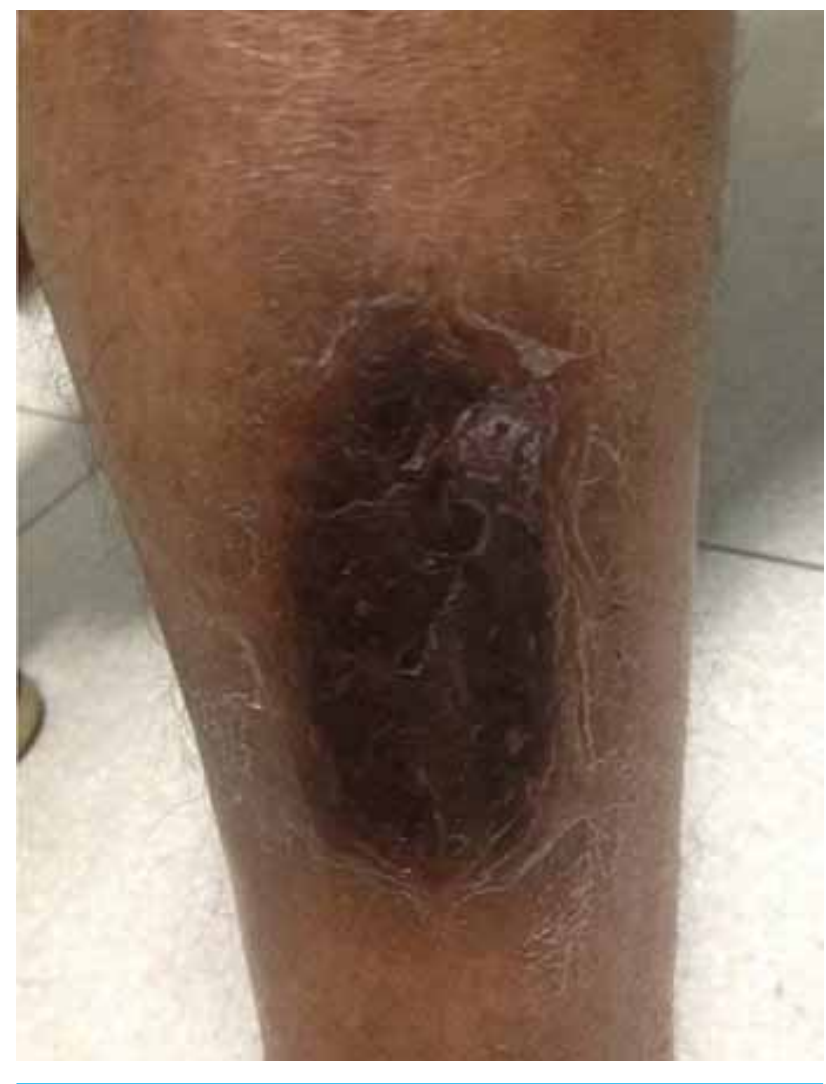

Figura 1: Perna esquerda (face anterior) - placa eritematoacastanhada infiltrativa descamativa.

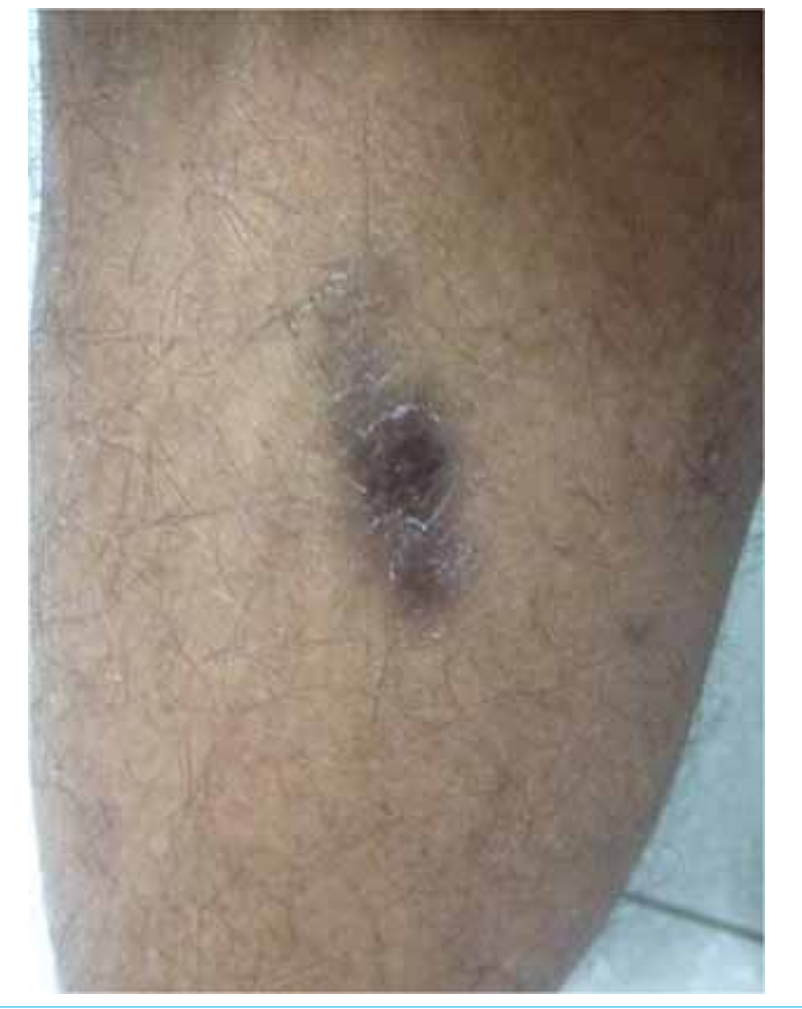

Figura 2: Perna esquerda (face posterior) - lesão menor. 


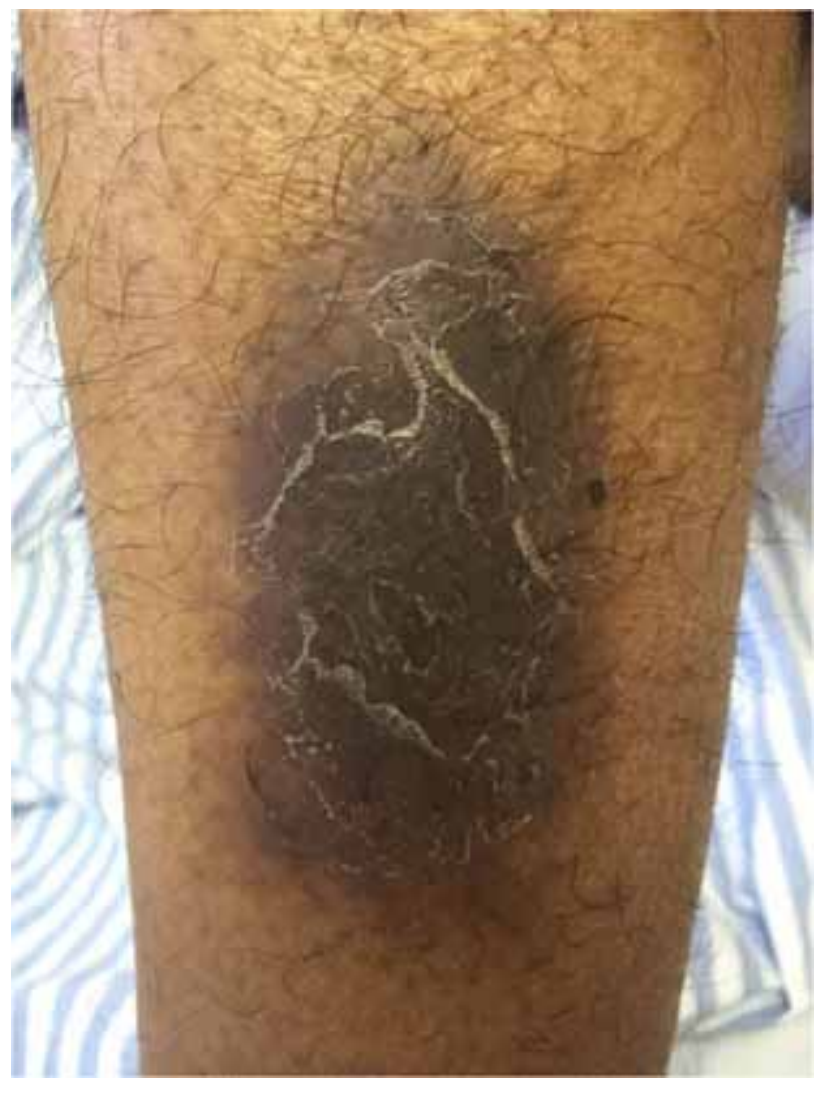

Figura 3: Perna direita, face anterior - lesão semelhante à perna esquerda.

metros, e com distribuição multifocal, acometendo também, na metade dos pacientes, mucosa oral e trato gastrointestinal. ${ }^{6} \mathrm{Com}$ a evolução, as lesões tornam-se endurecidas, acastanhadas e com superfície irregular, podendo ulcerar e apresentar edema perilesional. ${ }^{12}$ Têm preferência pelas localizações na cabeça, no pescoço e no tronco, podendo variar de poucas lesões isoladas a centenas de lesões disseminadas.

O diagnóstico diferencial inclui angiomatose bacilar, linfoma cutâneo, acroangiodermatite, histiocitoma fibroso aneurismático, entre outros. ${ }^{13}$

Infecção ativa por CMV acomete cerca de 90\% dos casos de pacientes soropositivos. É um dos agentes oportunistas mais comuns em pacientes infectados com HIV, sendo um dos grandes causadores de morbidade e mortalidade nesses pacientes. Os fatores de risco para o desenvolvimento da doença por CMV nesta população incluem o aumento do estágio de imunossupressão, manifestado pelo decréscimo da contagem de células $\mathrm{T} \mathrm{CD}^{+}{ }^{+}$e a presença da viremia por CMV. ${ }^{14}$

A infecção por CMV é frequente nos que apresentam risco para o desenvolvimento do sarcoma de Kaposi, por ativar HHV-8, aumentando a expressão dos genes líticos, e assim favorecer o aparecimento da neoplasia. ${ }^{15}$

A ativação do HHV-8 pelo CMV, presente em células infectadas latentemente pode gerar vírus capazes de semear as células circulantes com o HHV-8, aumentando o número de células por ele infectadas, e assim, contribuir para formação de tumores. ${ }^{15}$ Portanto, agentes de doenças infecciosas quando presentes em pacientes imunocomprometidos pode interagir e exacerbar a doença, por isso a importância de investigação de outras doenças infecciosas quando o paciente com aids se apresenta com sarcoma de Kaposi.

O maior interesse deste relato é enfatizar o importante aspecto, relatado na literatura e constatado neste caso, reafirmando que pacientes com sorologia positiva para HIV, que apresentam infecção pelo CMV, têm maior predisposição para o desenvolvimento de sarcoma de Kaposi. ${ }^{16}$

\section{REFERÊNCIA}

1-Ruiz MAG, Rivero JQ, García JLM, Rodríguez JJC, Kavadoy YR, Carmona MFR, et al. High-dose-rate brechytherapy in the treatment of skin Kaposi sarcoma. J Contemp Brachytherapy 2017; 9: 561-5.

2-Yenice MG, Varnalý E, ạeker KG, Kavak A, Tuðcu V. Scrotal Kaposi's Sarcoma in HIV-negative patient: A case report and review os the literature. Turk J Urol 2018; 44:182-4.

3-El-Mallawany NK, Kamiyango W, Slone JS, Villiera J, Kovarik CL, Cox CM et al. Clinical factors associated with long-term complete remission versus poor response to chemotherapy in HIV-infected children and adolescents with Kaposi sarcoma receiving bleomycin and vincristine: a retrospective observational study. PloS one 2016; 11: 1-17.

4-Akinde $\mathrm{O}$, Obadofin $\mathrm{O}$, Adeyemo T, Omoseebi O, Ikeri $\mathrm{N}$, Okonkwo I et al. Kaposi sarcoma among HIV infected patients in Lagos University Teaching Hospital, Nigeria: a 14-Year retrospective clinicopathological study. J Skin Cancer 2016; 2016(2016): 1-6.

5-De Barros CEP, Duarte SC, Shimoda E, Rodrigues CS, Cordeiro KF. Sarcoma de Kaposi como diagnóstico diferencial de lesões vulvares: relato de caso e revisão bibliográfica. Revista Cientifíca da FMC 2013; 8: 26- 30 .

6-Patrocínio J, Santo ARE, Patrocínio J, Pereira R, Gomes F, Louro F. Sarcoma de Kaposi, a propósito de um caso clínico. Rev Clin Hosp Prof Dr Fernando Fonseca. 2016; 4: 45-7. 
7-Kak I, Salama S, Gahla G, Naqvi A e Alowami S. A case of patch stage of Kaposi's sarcoma and discussion of the differential diagnosis. Rare tumors. 2016; 8: 30-2.

8 Luchetti AT, Porto LR, Moura C. Incidência de infecção por citomegalovírus em pacientes portadores de síndrome da imunodeficiência adquirida atendidos em Jundiaí, SP. J Health Sci Inst. 2015; 33: 21-5.

9 Silva CM, Peder LD, Deluca J, Horvath JD, Silva ES, Teixeira JJV, Bertolini DA et al. Soroprevalência de citomegalovírus em HIV positivos em um centro de referência no Brasil. Revista Saúde e Pesquisa. 2016; 9: 433-41.

10- Matos SB, Meyer R, Lima FWM. Citomegalovírus: uma revisão da patogenia, epidemiologia e diagnóstico da infecção. Rev. Saúde. 2011; 7: 44-57.

11-Santos M, Vilasboas V, Mendes L, Talhari C, Talhari S. Lymphangiectatic Kaposi's sarcoma in a patient with AIDS. An Bras Dermatol 2013; 88: 276-8.
12-Terra APS, Silva-Vergara ML, Gomes RAS, Pereira CLL, Simpson AJG, Caballero OL. Monitoramento de pacientes com AIDS para o desenvolvimento de doença por citomegalovirus (CMV) usando-se PCR multiplex. Rev. Soc. Bras. Med 2000; 33: 583-9.

13-Ohe, EMDN, Padilha MHVQ, Enokihara MMSS, Almeida FA, Porro AM. Sarcoma de Kaposi clássico fatal. An Bras Dermatol 2010; 85: 375-9.

14-Fonseca BAL, Bollela VR, Neto RJP. Sarcoma de Kaposi e síndrome da imunodeficiência adquirida: características desta associação, incluindo novos conceitos sobre patogênese e tratamento. Medicina (Ribeirão Preto) 1999; 32: 26-39.

15-Vieira J, O‘Hearn P, Kimball L, Chandran B, Corey L. Activation of Kaposi's sarcoma-associated herpesvirus (human herpesvirus 8 ) lytic replication by human cytomegalovirus. J Virol 2001; 75: 1378-86.

16-Leao JC, Hinrichsen SL, Freitas BL, Porter SR. Herpes vírus humano-8 e sarcoma de Kaposi. Rev Assoc Med Bras 1999; 45: 55-62. 